\title{
Predictors for procedural success and all-cause mortality in patients undergoing transcatheter mitral valve edge-to-edge repair for mitral regurgitation
}

\author{
Lukas Stolz ${ }^{1, \#}$, Martin Orban ${ }^{1,2, \#, ~ D a n i e l ~ B r a u n ~}{ }^{1}$, Michael Nabauer $^{1,2}$, Christian Hagl $^{3}$, Steffen Massberg $^{1,2}$, \\ Jörg Hausleiter ${ }^{1,2, *}$, Mathias Orban ${ }^{1,2, *}$ \\ 'Department of Cardiology, Klinikum der Universität München - Medizinische Klinik und Poliklinik I, Munich 81377, Germany. \\ ${ }^{2}$ Department of Cardiology, Munich Heart Alliance - Partner Site German Center for Cardiovascular Disease (DZHK), Munich \\ 81377, Germany. \\ ${ }^{3}$ Department of Cardiac Surgery, Klinikum der Universität München - Herzchirurgische Klinik und Poliklinik, Munich 81377, \\ Germany. \\ \#These authors contributed equally. \\ *These senior authors contributed equally.
}

Correspondence to: Dr. Mathias Orban, Medizinische Klinik und Poliklinik I, Ludwig-Maximilians Universität München, Marchioninistraße 15, München D 81377, Germany. E-mail: morban@med.Imu.de

How to cite this article: Stolz L, Orban M, Braun D, Nabauer M, Hagl C, Massberg S, Hausleiter J, Orban M. Predictors for procedural success and all-cause mortality in patients undergoing transcatheter mitral valve edge-to-edge repair for mitral regurgitation. Mini-invasive Surg 2020;4:76. http://dx.doi.org/10.20517/2574-1225.2020.69

Received: 6 Jul 2020 First Decision: 10 Aug 2020 Revised: 17 Sep 2020 Accepted: 24 Sep 2020 Published: 6 Nov 2020

Academic Editor: Azeem Latib Copy Editor: Cai-Hong Wang Production Editor: Jing Yu

\begin{abstract}
A growing body of evidence shows that transcatheter mitral valve edge-to-edge repair (TMVr) for mitral regurgitation (MR) improves symptoms and prognosis of patients with heart failure. Still, as recently shown by two large randomized controlled trials (COAPT and MITRA-FR), there is differing information on which patients have the largest benefit. We aimed to summarize the current knowledge of clinical and anatomic predictors for acute procedural failure and long-term all-cause mortality after TMVr. TMVr is an effective treatment option for patients with symptomatic MR fulfilling certain echocardiographic and clinical criteria or being ineligible for surgery despite optimal medical therapy. Acute procedural failure is influenced by anatomic features of the mitral valve, among those are increased tenting and mitral valve leaflet configuration, leaflet-to-annulus index, as well as the mitral valve opening area. In contrast, anatomy of the mitral valve plays a minor role in predicting all-cause mortality after TMVr. This endpoint is associated with patient comorbidities (e.g., renal failure and chronic lung disease), severe heart failure as expressed by New York Hear Association functional class (NYHA) IV, left and right heart dysfunction, laboratory parameters (NT-proBNP), clinical scoring systems (STS and EuroScore), and procedural MR reduction. In patients undergoing $T M V r$ for severe MR, careful preprocedural evaluation of relevant
\end{abstract}

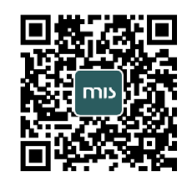


comorbidities, mitral valve anatomy, as well as left and right heart function can provide detailed prognostic value regarding acute procedural success and long-term survival.

Keywords: MitraClip, transcatheter mitral valve edge-to-edge repair, predictors for mortality, secondary mitral regurgitation, primary mitral regurgitation, heart failure, percutaneous mitral valve repair

\section{INTRODUCTION}

Mitral regurgitation (MR) is a major contributor to cardiovascular morbidity and mortality in patients with heart failure ${ }^{[1-3]}$. With more than ten years of clinical experience and continuous technical development, transcatheter mitral valve edge-to-edge repair (TMVr) is a well-established treatment option for patients suffering from primary (PMR) or secondary (SMR) mitral regurgitation. In PMR patients, structural damage of different parts of the valvular apparatus itself can lead to development of MR, while SMR is caused by atrial and ventricular pathologies ${ }^{[4]}$. Accordingly, PMR and SMR themselves form heterogeneous groups and can occur in combination, a fact which must be taken into account for therapeutic decisions and device selection ${ }^{[5]}$. In PMR, TMVr is recommended in case of prohibitive surgical risk and absence of adverse anatomic features, based on the results of the EVEREST (Endovascular Valve Edge-to-Edge Repair Study) trials ${ }^{[6-8]}$. For SMR, the 2020 Focused Update of the 2017 ACC Expert Consensus Decision Pathway on the Management of Mitral Regurgitation does not include surgical ineligibility as a primary criterium for TMVr usage. TMVr can be the therapy of choice for severe SMR with left ventricular ejection fraction (LV-EF) between $20 \%$ to $50 \%$, left ventricular end diastolic diameter (LV-EDD) $<7.0 \mathrm{~cm}$, and persistence of clinical signs and symptoms of heart failure despite of optimal guideline-recommended medical treatment (GDMT) and, if applicable, cardiac resynchronization therapy ${ }^{[8-11]}$. These recommendations are based on two large randomized-controlled trials (Cardiovascular Outcomes Assessment of the MitraClip Percutaneous Therapy for Heart Failure Patients with Functional Mitral Regurgitation - COAPT and Multicentre Study of Percutaneous Mitral Valve Repair MitraClip Device in Patients With Severe Secondary Mitral Regurgitation - MITRA-FR), which revealed different findings regarding the prognostic benefit of TMVr treatment on top of GDMT in SMR patients ${ }^{[12,13]}$. Recently, several theories have been proposed to deliver potential explanations for these varying results ${ }^{[14-19]}$. Undoubtedly, patient selection for $\mathrm{TMVr}$ could be a crucial factor influencing not only clinical outcome but also procedural success. The influence of cardiac anatomic parameters on outcome after TMVr is less understood, but has gained recent attention to optimize procedural and clinical results ${ }^{[20]}$. In this review, we evaluate the current data on the impact of anatomical and functional left and right heart features, as well as clinical parameters and comorbidities on acute procedural success/failure and mortality after TMVr.

\section{ENDPOINTS AND PATIENT COHORT}

The two common endpoints in outcome analysis after TMVr that this article focuses on are acute procedural failure (APF) and all-cause and/or cardiac mortality ${ }^{[21-24]}$. The Mitral Valve Academic Research Consortium (MVARC) differentiates between technical, device, procedural, and patient success ${ }^{[24]}$. In other words, APF is the absence of procedural success, which consists of technical success at exit from the catheterization laboratory, absence of procedural mortality or stroke, and reduction to MR $2+$ or lower ${ }^{[24]}$. Clearly, reasons for APF could be inability of device implantation due to individual anatomical features or generation of significant mitral valve stenosis represented by increasing mean mitral valve pressure gradients (MV mean PG). MVARC recommends postprocedural MV mean PG not to exceed $5 \mathrm{mmHg}^{[24]}$. Secondly, APF can be caused by insufficient MR reduction despite successful implantation of the device. According to MVARC criteria, procedural results are defined as optimal in case of absent or trace postprocedural $\mathrm{MR}^{[24]}$. 
PMR and SMR are two pathophysiologically different entities of mitral valve disease which both lead to similar clinical signs and symptoms. We believe that based on vast differences in baseline clinical characteristics, cardiac anatomy and function, baseline procedural risk before TMVr, and outcome after TMVr, patients with SMR and PMR should be analyzed separately ${ }^{[25]}$. This viewpoint is supported by an increasing body of evidence. Nevertheless, the majority of registries have reported on cohorts of both PMR and SMR without dedicated analysis of separate entities. Therefore, this review divides each section by MR sub-collectives (composed PMR and SMR, PMR only, and SMR only collectives).

\section{PREDICTING PROCEDURAL SUCCESS AND FAILURE IN PATIENTS UNDERGOING TMVR FOR}

\section{MR}

Comprehensive, unambiguous analysis of procedural success and failure is hindered by varying definitions in studies on TMVr. Albeit effective MR reduction is feasible in both PMR and SMR, some TMVr studies suggest more profound MR reduction in patients with $\mathrm{PMR}^{[26]}$, while some report higher rates of APF in $\mathrm{PMR}^{[27]}$ and some did not find any differences ${ }^{[28]}$. Comparisons between procedural MR reduction in patients with PMR and patients with SMR are further complicated by different definitions of MR severity and challenging assessment of quantitative MR parameters after device placement.

\section{Composed PMR and SMR patient collective}

Dörr et al. ${ }^{[29]}$ identified BNP levels and two biomarkers of cardiac fibrotic alterations, galectin-3 (Gal-3) and suppression of tumorigenicity 2 (ST2), as predictors for successful MR reduction by $\geq 2$ grades. It can be assumed that patients with higher levels of Gal-3 and ST2 are in a more advanced state of heart failure with ongoing fibrotic damage. This may alter the cardiac response to TMVr treatment, hinder reverse remodeling, and result in worse procedural outcomes.

Furthermore, Thaden et al. ${ }^{[30]}$ sought to determine predictors of hemodynamic success, which was defined as at least $40 \%$ reduction of left atrial V wave compared to baseline. Multivariable analysis revealed flail scallop [Figure 1A], single jet or multiple jets originating from a single scallop [Figure 1B], and good or excellent three-dimensional image quality as independent predictors for hemodynamic success. Besides that, preprocedural MV mean PG, mitral annular calcification, and deployment of more than one clip predicted development of mitral stenosis with a mean gradient greater than $5 \mathrm{mmHg}$.

\section{PMR only collective}

Detailed three-dimensional (3D) analysis of the MV can help to identify predictors for optimal MR reduction after TMVr. In PMR, low MV leaflet tenting volume [Figure 1C] and height [Figure 1D] were predictive of optimal MR reduction ${ }^{[31]}$. Even in the case of Carpentier classification type II PMR with prolapse of leaflet, concomitant regional tenting patterns may complicate optimal MR reduction ${ }^{[31]}$. Another $3 \mathrm{D}$ analysis found a novel predictive parameter called MV leaflet-to-annulus index (LAI), defined as the ratio of the sum of the anterior and posterior MV leaflet and the anteroposterior mitral annular length [Figure 1E]. Low LAI indicates a leaflet-to-annulus disproportionality and significantly predicts residual $\mathrm{MR}$ after $\mathrm{TMVr}^{[32]}$. Identifying patients with inadequate MR reduction is important as relevant residual MR is associated with worse survival rates in several studies ${ }^{[33-37]}$.

Besides these predictors for residual MR, development of postprocedural mitral stenosis can lead to APF. Two predictors of a MV mean PG $\leq 4 \mathrm{mmHg}$ after clip deployment in PMR were preprocedural mitral valve opening area of $(\mathrm{MVOA}) \geq 3.94 \mathrm{~cm}^{2}$ and medial-lateral diameter of left ventricle (LV) inflow orifice $\geq$ $3.23 \mathrm{~cm}$ for patients receiving one implanted clip. In case of two clips, cut-offs were $4.82 \mathrm{~cm}^{2}$ and $3.29 \mathrm{~cm}$, respectively ${ }^{[38]}$. 

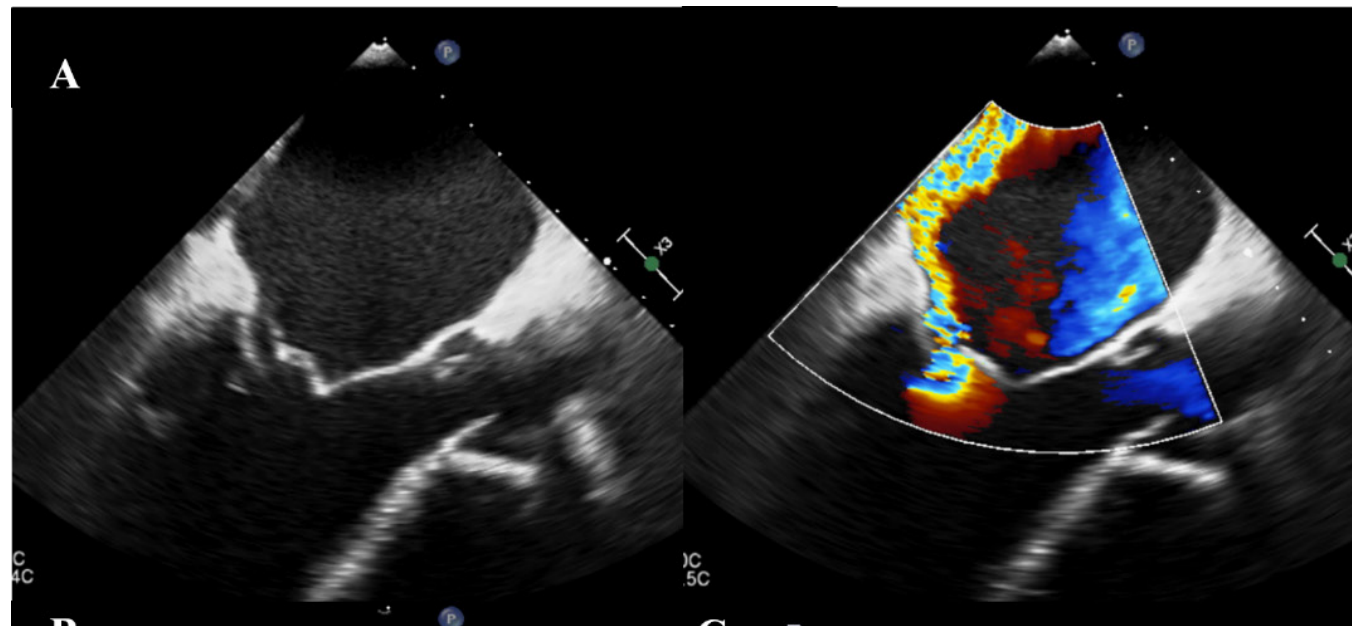

B C $A$
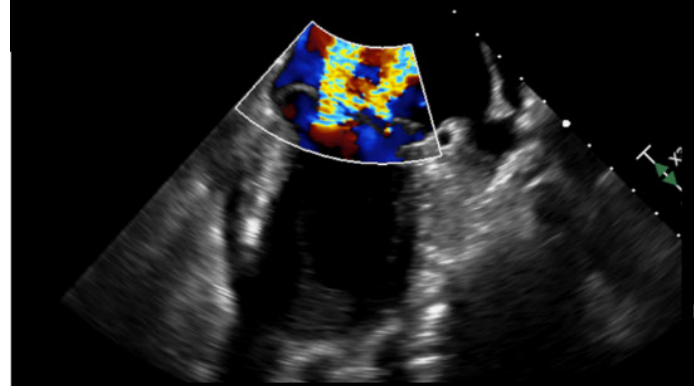

D $\mathbf{A}$

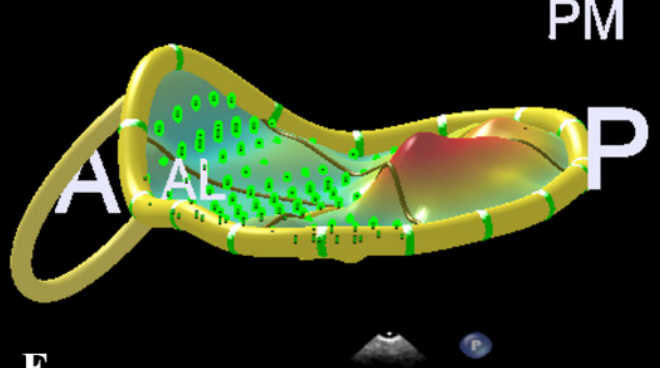

$\mathbf{E}$
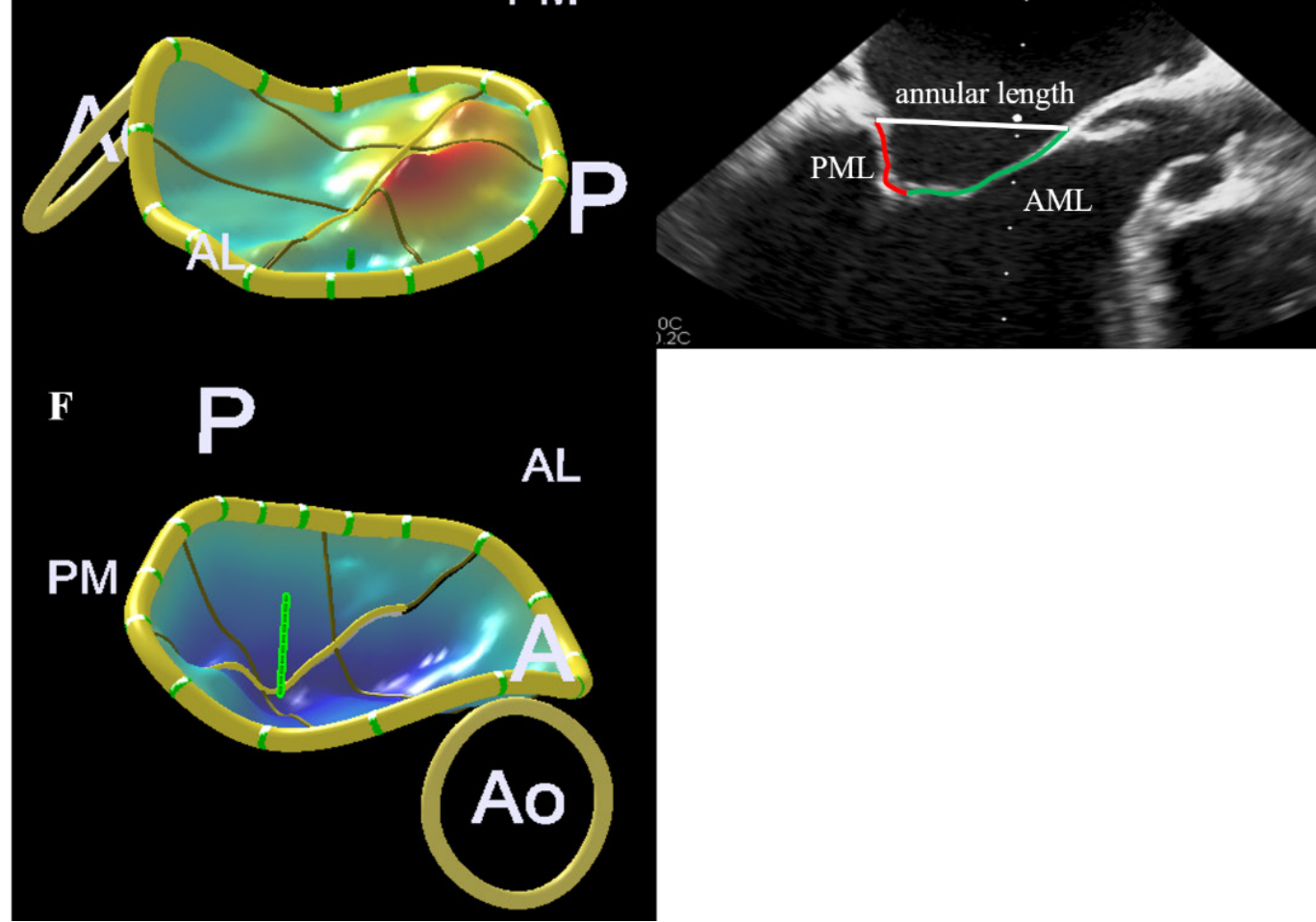

Figure 1. Anatomic predictors for procedural success and failure after TMVr. A: flail scallop is associated with worse hemodynamic success after TMVr in primary mitral regurgitation patients; B: A single or multiple jets originating from one flail scallop are associated with hemodynamic success after $\mathrm{TMVr}$; lower tenting volume (C) and tenting height (D) are associated with optimal MR reduction; E: low mitral valve leaflet-to-annulus index predicts residual MR; F: increasing annular height predicts optimal MR reduction. A: anterior; P: posterior; AL: anterolateral; PM: posteromedial; Ao: aortic valve; PML: posterior mitral valve leaflet; AML: anterior mitral valve leaflet; TMVr: transcatheter mitral valve edge-to-edge repair; MR: mitral regurgitation 
Table 1. Left heart: predictors for all-cause mortality after TMVr for MR

\begin{tabular}{llll}
\hline Parameter & Cut-off & MR etiology & Ref. \\
\hline LV-EF & $\leq 25 \%$ & SMR & {$[48]$} \\
& $<27 \%$ & SMR & {$[46]$} \\
& $<30 \%$ & SMR & {$[43]$} \\
Stroke volume & $<30 \%$ & SMR/PMR & {$[35,42,57,76]$} \\
LV dysfunction with and without CAD & $\star$ & SMR/PMR & {$[25,28]$} \\
LV-EDV & $*$ & PMR & {$[45]$} \\
LV-EDD & $* *$ & PMR & {$[45]$} \\
& $>216 \mathrm{~mL}$ & SMR & {$[48]$} \\
Afib & $*$ & SMR & {$[49]$} \\
& $*$ & SMR/PMR & {$[44]$} \\
LA-EF change & $* *$ & SMR & {$[47,48]$} \\
LA diameter & $* *$ & SMR/PMR & {$[51]$} \\
\hline
\end{tabular}

${ }^{\star}$ Continuous parameter; ${ }^{\star \star}$ binary parameter; ${ }^{\star \star \star}$ cardiac death. TMVr: transcatheter mitral valve edge-to-edge repair; MR: mitral regurgitation; SMR: secondary mitral regurgitation; PMR: primary mitral regurgitation; LV-EF: left ventricular ejection fraction; CAD: coronary artery disease; LV-EDV: left ventricular end diastolic volume; LV-EDD: left ventricular end diastolic diameter; Afib: atrial fibrillation; LA-EF: left ventricular ejection fraction; LA: left atrium

\section{SMR only collective}

For SMR, anatomic parameters of the mitral valve that could influence procedural success are mainly determined by atrio-ventricular architecture since leaflets do not have structural damage by definition. Several anatomic configurations of the MV are associated with optimal MR reduction by TMVr: Among those are increasing annular height $[\text { Figure } 1 \mathrm{~F}]^{[31]}$, less planar MV anatomy ${ }^{[31,39-41]}$ and, alike in PMR, the LAI parameter ${ }^{[32]}$. Stolfo et al. ${ }^{[41]}$ identified left ventricular end diastolic volume index and anteroposterior mitral annulus diameter as independent predictors for device failure according to MVARC criteria ${ }^{[24,4]}$. More severe dilation of the left ventricle leads to flattening deformation of the MV apparatus complicating TMVr procedure, while larger mitral annulus diameters impair proper leaflet coaptation.

Comparable to PMR, preprocedural MVOA and medial-lateral diameter of LV inflow orifice can also predict postprocedural mitral stenosis. For one and two clips, the cut-off values were $3.77 \mathrm{~cm}^{2} / 5.05 \mathrm{~cm}^{2}$ and $3.03 \mathrm{~cm} / 3.39 \mathrm{~cm}$, respectively ${ }^{[38]}$.

\section{PREDICTING ALL-CAUSE MORTALITY IN PATIENTS UNDERGOING TMVR FOR MR}

Within the last ten years, several reports aimed at identifying predictors for all-cause mortality in patients with MR after TMVr. Most of these studies were based on a composed collective of patients with PMR and SMR, while large, dedicated data sets for SMR and especially PMR alone are rare.

\section{LEFT VENTRICULAR FUNCTION AND DIMENSIONS}

\section{Composed PMR and SMR patient collective}

One of the main predictors for all-cause mortality in patients with MR undergoing TMVr is impairment of left ventricular function, represented by reduced LV-EF. Several analyses identified impaired LV-EF as highly predictive for five-year ${ }^{[28,35]}$ and long-term mortality ${ }^{[25,42,43]}$ [Table 1]. Surprisingly, left ventricular size and geometry do not seem to play a major role in predicting TMVr all-cause mortality when including both SMR and PMR patients into multivariable models. Only one study specifically focusing on cardiac mortality reported increased LV-EDD as a significant predictor ${ }^{[4]}$ [Table 1].

\section{PMR only collective}

In patients with PMR, impaired left ventricular stroke volume and LV-EF are predictors for all-cause mortality [Table 1 $]^{[4]}$. 


\section{SMR only collective}

Consistently, impaired LV-EF leads to significantly worsened long-term survival in patients with SMR after $\mathrm{TMVr}^{[25,46-48]}$. In contrast to mixed cohort analysis, severe LV dilatation, measured either by LV-EDD ${ }^{[49]}$ or left ventricular end diastolic volume ${ }^{[48]}$, was identified as a predictor for all-cause mortality in patients with SMR [Table 1]. After publication of the COAPT and MITRA-FR trials, a discussion about possible reasons for the diverging prognostic results has evolved and several explanations have been proposed. Among them are operator experience, intensity of concomitant medical therapy, progression of heart failure at baseline, and procedural MR reduction. Since mean left ventricular end diastolic volume was very high in MITRA-FR, patients in this trial might have had end-stage heart failure with severe LV dilatation. The proportionality of MR severity to LV dilatation, quantified by ratio of effective regurgitant orifice area to $\mathrm{LV}$ end diastolic volume, has recently gained attention ${ }^{[1,50]}$. Latest analyses showed that the proportionality concept as a prognostic framework might be applicable to medically treated SMR patients. Its influence on prognosis in TMVr-treated patients is probably less important, as TMVr effectively reduces MR and thus abolishes one component of the proportionality equation ${ }^{[17,19]}$.

\section{LEFT ATRIAL FUNCTION AND DIMENSIONS}

\section{Composed PMR and SMR patient collective}

Atrial fibrillation or absence of sinus rhythm, as indicators of impaired LA function in addition to LA dilation, are linked to worse TMVr survival ${ }^{[42,51,52]}$. Severe LA dilatation with a diameter $\geq 55 \mathrm{~cm}$ seems to be a highly predictive cut-off value ${ }^{[52]}$. In contrast, improvement of LA ejection fraction from baseline to short term follow up (three to six month) is associated with lower all-cause long-term mortality ${ }^{[53]}$ [Table 1].

\section{SMR only collective}

While dedicated data for PMR patients are missing, atrial fibrillation ${ }^{[47,48]}$ and increased LA volume ${ }^{[47]}$ are associated with impaired long-term survival in SMR patients [Table 1]. Left atrial dysfunction in SMR patients recently gained attention as this condition can lead to MR in absence of severe systolic LV dysfunction. This pathology called atrial secondary mitral regurgitation (ASMR) is caused by either atrial fibrillation or heart failure with preserved ejection fraction (HFpEF), as both increase LA pressure and volume leading to annular flattening and alteration of left ventricular atrioventricular hemodynamics ${ }^{[54]}$. As HFpEF patients with SMR were excluded from large controlled randomized trials (COAPT or MITRA$\mathrm{FR})^{[12,13]}$, but undergo TMVr procedure in real-world clinical practice, impact of ASMR on survival and procedural success warants further investigation.

\section{MITRAL VALVE ANATOMY, HEMODYNAMICS, AND PROCEDURAL SUCCESS Composed PMR and SMR patient collective}

Elevated MV mean PG was identified as highly predictive in terms of all-cause mortality, both for preprocedural and postprocedural measurements. TMVr increases MV mean PG by reduction of mitral valve opening area ${ }^{[27,36,55]}$ [Table 2]. Additionally, previous MV surgery has been reported to have negative influence on long-term outcome ${ }^{[56]}$. Success of TMVr procedure itself is crucial for reduction of long-term mortality and reflects the benefit of this interventional approach on MR treatment. Absence of procedural $M R$ reduction and residual MR after procedure lead to severely impaired long-term survival ${ }^{[2,35,36,56-60]}$ [Table 2].

\section{PMR only collective}

In PMR patients, postprocedural MV mean PG is a significant predictor for survival ${ }^{[61]}$ [Table 2].

\section{SMR only collective}

Similar to findings in the composed SMR/PMR collective, residual SMR is a major factor contributing to mortality following TMVr procedure ${ }^{[33,49]}$. In particular, postprocedural MR vena contracta area is 
Table 2. Mitral valve: predictors for all-cause mortality after TMVr for MR

\begin{tabular}{|c|c|c|c|}
\hline Parameter & Cut-off & MR etiology & Ref. \\
\hline MV mean PG (pre) & $>1.5 \mathrm{mmHg}$ & $\begin{array}{l}\text { SMR/PMR } \\
\text { SMR/PMR }\end{array}$ & $\begin{array}{l}{[27]} \\
{[34,36]}\end{array}$ \\
\hline MV mean PG (post) & $\begin{array}{l}>5 \mathrm{mmHg} \text { (invasive) } \\
>4.4 \mathrm{mmHg} \text { (echo) }\end{array}$ & $\begin{array}{l}\text { SMR/PMR } \\
\text { SMR/PMR }\end{array}$ & $\begin{array}{l}{[55]} \\
{[55]}\end{array}$ \\
\hline Acute procedural failure & 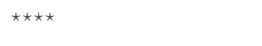 & $\mathrm{SMR} / \mathrm{PMR}$ & {$[57,76,87]$} \\
\hline Residual MR & $\begin{array}{l}\geq 2+ \\
\geq 2+ \\
\geq 3+ \\
\geq 2+ \\
\geq 3+ \\
\star \\
\star\end{array}$ & $\begin{array}{l}\text { SMR } \\
\text { SMR } \\
\text { SMR } \\
\text { SMR/PMR } \\
\text { SMR/PMR } \\
\text { SMR/PMR } \\
\text { SMR/PMR }\end{array}$ & $\begin{array}{l}{[33]} \\
{[49]} \\
{[33]} \\
{[32,58]} \\
{[59]} \\
{[25,34,36,56]} \\
{[44]}\end{array}$ \\
\hline MR recurrence $<2$ years & $\geq 2+$ & SMR/PMR & {$[35]$} \\
\hline VCA (post) & $>25 \mathrm{~mm}^{2}$ & SMR & {$[62]$} \\
\hline Previous MV surgery & $\star \star$ & $\mathrm{SMR} / \mathrm{PMR}$ & {$[56]$} \\
\hline
\end{tabular}

${ }^{\star}$ Continuous parameter; ${ }^{\star \star}$ binary parameter; ${ }^{\star \star \star}$ cardiac death; ${ }^{\star \star \star \star}$ operator-reported failure, conversion to surgery, abortion of procedure or severe residual mitral regurgitation. TMVr: transcatheter mitral valve edge-to-edge repair; MR: mitral regurgitation; SMR: secondary mitral regurgitation; PMR: primary mitral regurgitation; PG: pressure gradient; VCA: vena contracta area; MV: mitral valve

associated with worse long-term outcome ${ }^{[62]}$. Mitral valve anatomy itself seems to play a minor role in predicting long-term mortality after TMVr [Table 2]. The only MV configuration that impairs outcome in terms of higher MR severity at follow-up examination seems to be restricted posterior mitral valve leaflet motion defined as posterior mitral valve leaflet tethering angle $>45^{\circ[63]}$. Whether posterior mitral valve leaflet tethering impacts not only procedural success but also long-term mortality has not been shown so far.

The prognostic role of ischemic origin of SMR has been studied by several groups. Apparently, predictors for all-cause mortality could be different in ischemic versus non-ischemic SMR ${ }^{[64]}$. Tricuspid annular plane excursion, renal failure, diabetes mellitus, previous heart surgery, and coronary artery bypass graft are predictive for all-cause mortality in ischemic, but not in non-ischemic SMR ${ }^{[64]}$. Besides ischemic and non-ischemic subgroups, SMR etiologies can be separated by LV-LA function. Among those is ASMR, as previously mentioned ${ }^{[54,65,66]}$. While our knowledge of anatomy and pathophysiology of ASMR is growing, specific predictors for all-cause mortality after TMVr are so far lacking ${ }^{[66]}$.

\section{RIGHT VENTRICULAR FUNCTION AND PULMONARY HYPERTENSION Composed PMR and SMR patient collective}

Data on right ventricular (RV) dysfunction in composed PMR/SMR collectives are absent. Nevertheless, pulmonary hypertension has been shown to impair prognosis as it is associated with worse long-term survival ${ }^{[27,67-69]}$. Cut-off values for systolic pulmonary artery pressure as a measurement of pulmonary hypertension vary between $37 \mathrm{mmHg}$ and $60 \mathrm{mmHg}^{[67]}$ [Table 3].

\section{SMR only collective}

In contrast to the lack of data for PMR patients, there is a growing body of knowledge that RV dysfunction and pulmonary hypertension in SMR patients are crucial factors for the prognosis after TMVr, pulmonary hypertension (as expressed by elevated systolic pulmonary artery pressure) is also associated with longterm mortality in SMR patients [Table 3] ${ }^{[4]}$. Obviously, pulmonary hypertension due to left ventricular and atrial dysfunction and RV function are closely linked. Presence of RV dysfunction, as expressed by impaired tricuspid annular plane excursion or RV peak systolic velocity, leads to biventricular failure [Table 3] $]^{[47,70-72]}$. Importantly, TMVr treatment is capable of improving RV function. One study found an improvement of tricuspid annular plane excursion by $4 \mathrm{~mm}$ and peak systolic velocity by $4 \mathrm{~cm} / \mathrm{s}$ at 6 months follow up ${ }^{[73]}$. 
Table 3. Pulmonary system and right heart: predictors for all-cause mortality after TMVr for MR

\begin{tabular}{llll}
\hline Parameter & \multicolumn{1}{c}{ Cut-off } & MR etiology & Ref. \\
\hline CLD & $\star \star$ & SMR/PMR & {$[83]$} \\
SPAP & $>50 \mathrm{mmHg}$ & SMR & {$[88]$} \\
& $\star$ & SMR & {$[49]$} \\
& $>45 \mathrm{mmHg}$ & SMR/PMR & {$[87]$} \\
& $>50 \mathrm{mmHg}$ & SMR/PMR & {$[27]$} \\
& $37-50 \mathrm{mmHg}$ & SMR/PMR & {$[67]$} \\
& $>60 \mathrm{mmHg}$ & SMR/PMR & {$[37]$} \\
RVSP & $\star$ & SMR/PMR & {$[78]$} \\
PSV (DTI) & $<9.5 \mathrm{~cm} / \mathrm{s}$ & SMR & {$[72]$} \\
TAPSE & $<15 \mathrm{~mm}$ & SMR & {$[71]$} \\
& $\leq 16 \mathrm{~mm}$ & SMR & {$[70]$} \\
\hline
\end{tabular}

${ }^{\star}$ Continuous parameter; ${ }^{\star \star}$ binary parameter; ${ }^{\star \star \star}$ cardiac death. TMVr: transcatheter mitral valve edge-to-edge repair; MR: mitral regurgitation; SMR: secondary mitral regurgitation; PMR: primary mitral regurgitation; CLD: chronic lung disease; SPAP: systolic pulmonary artery pressure; PSV: peak systolic velocity; DTI: doppler tissue imaging; TAPSE: tricuspid annular plane systolic; RVSP: right ventricular systolic pressure

Table 4. Tricuspid and aortic valve: predictors for all-cause mortality after TMVr for MR

\begin{tabular}{llll}
\hline Parameter & Cut-off & MR etiology & Ref. \\
\hline TR (pre) & $\star$ & SMR & {$[77]$} \\
TR (post) & $\geq 3+$ & SMR/PMR & {$[37,56,57,74,76,87]$} \\
Previous AoV intervention & $\star$ & SMR & {$[43]$} \\
Moderate AR & $\star \star$ & SMR/PMR & {$[42,57,76,87]$} \\
\hline
\end{tabular}

*Ordinal parameter; ${ }^{*}$ binary parameter. TMVr: transcatheter mitral valve edge-to-edge repair; MR: mitral regurgitation; SMR: secondary mitral regurgitation; PMR: primary mitral regurgitation; TR: tricuspid regurgitation; AoV: aortic valve; AR: aortic regurgitation

\section{CONCOMITANT TRICUSPID AND AORTIC VALVE DISEASE}

\section{Composed PMR and SMR patient collective}

The relevance of concomitant valve disease in patients treated with TMVr has been shown for tricuspid and aortic valve regurgitation. Severity of pre- and postprocedural moderate or severe tricuspid regurgitation (TR) has repeatedly been shown as an important factor worsening long-term mortality [Table 4$]^{[37,42,43,56,74]}$. Whether TR contributes alone to dismal outcome or only in conjunction with RV dysfunction is controversial and has to be further assessed. In addition, a recent study reported about the negative impact of moderate aortic regurgitation on survival [Table 4$]^{[75]}$. Prior intervention of the aortic valve has repeatedly been reported as a negative prognostic factor for patients treated with $\mathrm{TMVr}^{[42,57,76]}$.

\section{SMR only collective}

Data about the role of concomitant TR in SMR patients are ambiguous, while again dedicated data of concomitant valvular pathology for PMR patients undergoing TMVr is unknown. While some authors found preprocedural severe TR as a predictor worsening prognosis after $\mathrm{TMVr}^{[77]}$ others, including the large COAPT trial with echocardiographic core lab assessment, did not ${ }^{[25,78]}$ [Table 4]. We believe that moderate or severe TR in patients with SMR is tightly connected to the prevalent biventricular failure, thus a bystander. Whether isolated TR in the absence of RV dysfunction might yield prognostic value in TMVrSMR patients has yet to be shown.

\section{RENAL FUNCTION}

Undoubtedly shown by a multitude of studies, impaired kidney function (defined as either reduced estimated glomerular filtration rate, elevated levels of creatinine or need of dialysis) is one of the strongest predictors for all-cause mortality in TMVr-treated patients ${ }^{[25,27,28,32,33,42,43,53,79,80]}$. Those findings are consistent in PMR, 
Table 5. Renal function: predictors for all-cause mortality after TMVr for MR

\begin{tabular}{llll}
\hline Parameter & \multicolumn{1}{c}{ Cut-off } & MR etiology & Ref. \\
\hline GFR & $<30 \mathrm{~mL} / \mathrm{min}$ & $\mathrm{SMR}$ & {$[72]$} \\
& $30-60 \mathrm{~mL} / \mathrm{min}$ & $\mathrm{SMR}$ & {$[43]$} \\
& $<50 \mathrm{~mL} / \mathrm{min}$ & $\mathrm{SMR}$ & {$[62]$} \\
& $\star$ & $\mathrm{SMR}$ & {$[53]$} \\
& $\star$ & $\mathrm{SMR} / \mathrm{PMR}$ & {$[25,79]$} \\
& $<60 \mathrm{~mL} / \mathrm{min}$ & $\mathrm{SMR} / \mathrm{PMR}{ }^{\star \star *}$ & {$[44]$} \\
Creatinine & $<60 \mathrm{~mL} / \mathrm{min}$ & $\mathrm{SMR} / \mathrm{PMR}$ & {$[37,58]$} \\
& $>1.5 \mathrm{mg} / \mathrm{dL}$ & $\mathrm{SMR} / \mathrm{PMR}$ & {$[35,42,57,76,87]$} \\
& $>2 \mathrm{mg} / \mathrm{dL}$ & $\mathrm{SMR} / \mathrm{PMR}$ & {$[27]$} \\
Renal failure & $\star$ & $\mathrm{SMR} / \mathrm{PMR}$ & {$[83]$} \\
Cystatin C & $\star \star$ & $\mathrm{PMR}$ & {$[45]$} \\
NGAL & $1.7 \mathrm{mg} / \mathrm{dL}$ vs. $2.4 \mathrm{mg} / \mathrm{dL}]$ & {$[89]$} \\
\hline
\end{tabular}

${ }^{\star}$ Continuous parameter; ${ }^{\star \star}$ binary parameter; ${ }^{* \star \star}$ cardiac death; ${ }^{\star \star \star \star \star \star}$ survivors vs. non survivors. TMVr: transcatheter mitral valve edge-toedge repair; MR: mitral regurgitation; SMR: secondary mitral regurgitation; PMR: primary mitral regurgitation; GFR: glomerular filtration rate; NGAL: neutrophil gelatinase-associated lipocalin

Table 6. Parameters of heart failure: predictors for all-cause mortality after TMVr for MR

\begin{tabular}{llll}
\hline Parameter & \multicolumn{1}{c}{ Cut-off } & MR etiology & Ref. \\
\hline NYHA & $\star$ & SMR & {$[77]$} \\
& $\geq$ III & SMR & {$[86]$} \\
& IV & SMR/PMR & {$[80]$} \\
& IV & SMR/PMR & {$[44]$} \\
NT-proBNP & $\geq 10000 \mathrm{pg} / \mathrm{mL}$ & SMR/PMR & {$[37,57,58,60,76,85,87,90]$} \\
& Per $10^{3}$ increase & SMR & {$[48]$} \\
& Log & SMR (non-ischemic) & {$[64]$} \\
Log & SMR/PMR & {$[80]$} \\
Prior cardiac decompensation & $\geq 5000 \mu \mathrm{S} / \mathrm{L}$ & SMR/PMR & {$[85]$} \\
Prior cardiac hospitalization & $\star \star$ & SMR/PMR & {$[56]$} \\
Length of hospitalization & $\star \star$ & SMR/PMR & {$[35,42]$} \\
\hline
\end{tabular}

${ }^{\star}$ Ordinal parameter; ${ }^{* \star}$ binary parameter; ${ }^{\star \star \star}$ cardiac death. TMVr: transcatheter mitral valve edge-to-edge repair; MR: mitral regurgitation; SMR: secondary mitral regurgitation; PMR: primary mitral regurgitation; NYHA: New York Heart Association

SMR and composed PMR/SMR collectives. Reported cut-off values in terms of all-cause mortality range from $<60 \mathrm{~mL} / \mathrm{min}$ to $<30 \mathrm{~mL} / \mathrm{min}$ for GRF and $1.5 \mathrm{mg} / \mathrm{dL}$ to $2.0 \mathrm{mg} / \mathrm{dL}$ for creatinine levels [Table 5]. In a composed SMR/PMR collective, other laboratory parameters of kidney function including Cystatin C and neutrophil gelatinase-associated lipocalin were also associated with worse outcome after $\mathrm{TMVr}^{[81,82]}$ [Table 5].

\section{COMORBIDITIES AND HEART FAILURE-RELATED PARAMETERS}

Besides kidney function, a broad variety of clinical conditions and comorbidities are accompanied by worse survival rates. Among those are chronic lung disease ${ }^{[2,83]}$, heart failure as expressed by elevated levels of the natriuretic peptide NTpro-BNP ${ }^{[80,84]}$ or worse New York Hear Association functional class (NYHA) functional class ${ }^{[42,57,58,60,80]}$ [Table 6], anemia ${ }^{[28,85]}$, elevated mean arterial blood pressure ${ }^{[85]}$, impaired exercise capacity (six minute walk test) ${ }^{[74]}$, and peripheral artery disease ${ }^{[42]}$ [Table 7]. Integrating several of the aforementioned conditions and comorbidities, the Society of Thoracic Surgery (STS) score as well as the EuroScore (logistic and EuroScore II) have been shown to predict outcome after $\mathrm{TMVr}^{[35]}$. Reported cutoffs are $\geq 20$ for logistic EuroScore and $\geq 12$ for STS Score [Table 7]. As advanced age comes along with a higher burden of comorbidities ${ }^{[28,62,64,80,83,86]}$ and male patients entail a higher number of cardiac risk factors, these demographics diminish prognosis ${ }^{[53,78]}$ [Table 7].

\section{DOES THE "IDEAL" TMVR PATIENT EXIST?}

Taking into account the broad variety of cardiac and extracardiac conditions influencing outcome after TMVr, it seems difficult to identify the "ideal" patient for this procedure. Generally speaking, survival 
Table 7. Comorbidities, demographics and risk scores: predictors for all-cause mortality after TMVr for MR

\begin{tabular}{|c|c|c|c|}
\hline Parameter & Cut-off & MR etiology & Ref. \\
\hline PAD & $\begin{array}{l}\star \star \\
\star \star\end{array}$ & $\begin{array}{l}\text { SMR } \\
\text { SMR/PMR }\end{array}$ & $\begin{array}{l}{[77]} \\
{[57,76,87]}\end{array}$ \\
\hline Anemia & $\star \star$ & SMR/PMR & {$[28,57,76]$} \\
\hline $\mathrm{Hb}$ & * & SMR/PMR & {$[85]$} \\
\hline Blood transfusion & $\geq 2$ Units & SMR & {$[86]$} \\
\hline MAP & * & SMR/PMR & [85] \\
\hline Ischemic MR & $\star \star$ & SMR/PMR & {$[60]$} \\
\hline Peak $\mathrm{VO}_{2}$ & $\star$ & SMR & {$[47]$} \\
\hline Age & $\begin{array}{l}\star \\
\star \\
\star \\
\star \star\end{array}$ & 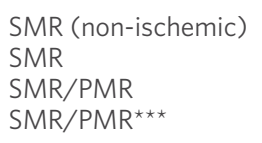 & $\begin{array}{l}{[64]} \\
{[86]} \\
{[62,83]} \\
{[28,80]}\end{array}$ \\
\hline Sex & 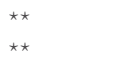 & $\begin{array}{l}\text { SMR } \\
\text { SMR/PMR }\end{array}$ & $\begin{array}{l}{[78]} \\
{[53]}\end{array}$ \\
\hline Log ES & $\begin{array}{l}\star \\
\star \\
\star \\
\star \\
>20 \\
\geq 20\end{array}$ & $\begin{array}{l}\text { SMR (ischemic) } \\
\text { SMR } \\
\text { SMR/PMR } \\
\text { SMR/PMR } \\
\text { SMR/PMR }\end{array}$ & $\begin{array}{l}{[64]} \\
{[45]} \\
{[80]} \\
{[80]} \\
{[36]}\end{array}$ \\
\hline STS & $\begin{array}{l}\star \\
\star \\
\geq 12 \\
\geq 12 \\
\end{array}$ & $\begin{array}{l}\text { SMR } \\
\text { SMR/PMR } \\
\text { SMR/PMR } \\
\text { SMR/PMR } \\
\end{array}$ & $\begin{array}{l}{[78]} \\
{[80]} \\
{[80]} \\
{[58]} \\
\end{array}$ \\
\hline
\end{tabular}

${ }^{\star}$ Continuous parameter; ${ }^{\star \star}$ binary parameter. TMVr: transcatheter mitral valve edge-to-edge repair; MR: mitral regurgitation; SMR: secondary mitral regurgitation; PMR: primary mitral regurgitation; PAD: peripheral artery disease; Hb: hemoglobin; MAP: mean arterial pressure; Peak $\mathrm{VO}_{2}$ : maximum oxygen uptake; log ES: logistic euroscore; STS: society of thoracic surgery risk score

prognosis correlates with the patient's overall health status, non-cardiac comorbidities, and most importantly, degree and characteristics of heart failure. This is intricate, as profound surgical risk and comorbidities often are the main reason for considering TMVr as primary therapy.

First and foremost, successful MR reduction by device implantation is the key for any clinical or prognostic improvement. Guided by proper two- and three-dimensional echocardiography, an experienced interventionalist is capable of achieving maximum procedural reduction of MR without generation of MV stenosis. Ideal prerequisites would be a low mean mitral valve pressure gradient, large mitral valve opening area, and wide LV inflow diameter. Furthermore, MV geometry, as influenced by left ventricular and atrial anatomy, should be preserved, without flattening of the MV annulus, lowering of the anterior mitral valve angle, or disproportionate leaflet-to annulus ratio. Furthermore, if there is a concomitant secondary component to PMR, tenting volume and height should be low.

In terms of survival, the ideal patient is believed to present with a minimal spectrum of extracardiac comorbidities, no concomitant aortic, tricuspid, or pulmonic valve pathologies, moderately impaired LV function, and absence of right ventricular failure and pulmonary hypertension. Generally speaking, after successful intervention, the patients' overall health status determines survival prognosis, while anatomic features seem to play a minor role for further prognosis.

\section{CONCLUSION}

With successful MR reduction rates of more than $95 \%$ in the majority of studies, the TMVr procedure for severe MR can be performed effectively and safely in a wide variety of mitral valve configurations with different underlying left heart diseases. For acute procedural failure, anatomic and hemodynamic parameters of the MV are important predictors. In contrast, clinical baseline characteristics, comorbidities, atrioventricular echocardiographic parameters, and procedural MR reduction are important for long-term prognosis. 
Since patients with PMR have severe structural pathologies of the MV, leaflet configuration seems to be more important compared to SMR patients. Therefore, we recommend stricter separation of SMR and PMR etiology within studies, as well as differentiating "sub-etiologies" of SMR (ischemic MR, non-ischemic MR, ASMR, and HFmrEF-SMR), which could be done by multi-center pooling of data. A prerequisite is comprehensive guideline-recommended echocardiographic assessments of cardiac anatomy and function, also including the right heart and pulmonary vasculature. This integrated approach is challenging but would facilitate further understanding of pathophysiology and outcome in a diversity of SMR subtypes undergoing TMVr, thereby improving patient selection and procedural MR reduction to achieve optimal outcome after TMVr.

\section{DECLARATIONS}

\section{Authors' contributions}

Drafting of conceptual and methodologic framework: Stolz L, Orban M, Hausleiter J, Orban M

Manuscript writing: Stolz L, Orban M, Orban M

Manuscript review: Braun D, Nabauer M, Hagl C, Massberg S, Hausleiter J

\section{Availability of data and materials}

Not applicable.

\section{Financial support and sponsorship}

This work was supported by Klinikum der Universität München.

\section{Conflicts of interest}

Martin Orban has received speaker honoraria from SedanaMedical, AstraZeneca and Bayer Vital. Michael Nabauer, Mathias Orban and Daniel Braun have received speaker honoraria from Abbott Vascular. Jörg Hausleiter has received speaker honoraria from Abbott Vascular and Edwards Lifesciences. The other authors declared that there are no conflicts of interest.

\section{Ethical approval and consent to participate}

Not applicable.

\section{Consent for publication}

Not applicable.

\section{Copyright}

(c) The Author(s) 2020.

\section{REFERENCES}

1. Asgar AW, Mack MJ, Stone GW. Secondary mitral regurgitation in heart failure: pathophysiology, prognosis, and therapeutic considerations. J Am Coll Cardiol 2015;65:1231-48.

2. Boudoulas KD, Vallakati A, Pitsis AA, Orsinelli DA, Abraham WT. The use of MitraClip in secondary mitral regurgitation and heart failure. Cardiovascular revascularization medicine: including molecular interventions. Cardiovasc Revasc Med 2020:S15538389(20)30262-1.

3. Goel K, Barker CM, Lindenfeld J. Contemporary management of secondary mitral regurgitation. Eur Cardiol 2020;15:e22.

4. Zoghbi WA, Adams D, Bonow RO, et al. Recommendations for noninvasive evaluation of native valvular regurgitation: a report from the American society of echocardiography developed in collaboration with the society for cardiovascular magnetic resonance. $J$ Am Soc Echocardiogr 2017;30:303-71.

5. Chehab O, Roberts-Thomson R, Ng Yin Ling C, et al. Secondary mitral regurgitation: pathophysiology, proportionality and prognosis. Heart 2020;106:716-23.

6. Feldman T, Kar S, Elmariah S, et al; EVEREST II Investigators. Randomized comparison of percutaneous repair and surgery for mitral regurgitation: 5-year results of EVEREST II. J Am Coll Cardiol 2015;66:2844-54. 
7. Glower DD, Kar S, Trento A, et al. Percutaneous mitral valve repair for mitral regurgitation in high-risk patients: results of the EVEREST II study. J Am Coll Cardiol 2014;64:172-81.

8. Bonow RO, O'Gara PT, Adams DH, et al. 2020 Focused Update of the 2017 ACC Expert Consensus Decision Pathway on the Management of Mitral Regurgitation: A Report of the American College of Cardiology Solution Set Oversight Committee. $J$ Am Coll Cardiol 2020;75:2236-70.

9. Baumgartner H, Falk V, Bax JJ, et al. 2017 ESC/EACTS Guidelines for the management of valvular heart disease. Eur Heart J 2017;38:2739-91.

10. Marmagkiolis K, Hakeem A, Ebersole DG, et al. Clinical outcomes of percutaneous mitral valve repair with MitraClip for the management of functional mitral regurgitation. Catheter Cardiovasc Interv 2019;94:820-6.

11. Praz F, Braun D, Unterhuber M, et al. Edge-to-edge mitral valve repair with extended clip arms: early experience from a multicenter observational study. JACC Cardiovasc Interv 2019;12:1356-65.

12. Obadia JF, Messika-Zeitoun D, Leurent G, et al; MITRA-FR Investigators. Percutaneous repair or medical treatment for secondary mitral regurgitation. N Engl J Med 2018;379:2297-306.

13. Stone GW, Lindenfeld J, Abraham WT, et al; COAPT Investigators. Transcatheter mitral-valve repair in patients with heart failure. $N$ Engl J Med 2018;379:2307-18.

14. Grayburn PA, Sannino A, Packer M. Proportionate and disproportionate functional mitral regurgitation: a new conceptual framework that reconciles the results of the MITRA-FR and COAPT trials. JACC Cardiovasc Imaging 2019;12:353-62.

15. Mauricio R, Kumbhani DJ. MitraClip: how do we reconcile the inconsistent findings of MITRA-FR and COAPT? Curr Cardiol Rep 2019;21:150.

16. Luc JGY, Hong JC, Cheung AW, et al. The MITRA-FR trial vs the COAPT trial: more complementary than contradictory? Ann Thorac Surg 2019;108:965-8.

17. Gaasch WH, Aurigemma GP, Meyer TE. An appraisal of the association of clinical outcomes with the severity of regurgitant volume relative to end-diastolic volume in patients with secondary mitral regurgitation. JAMA Cardiol 2020;5:476-81.

18. Adamo M, Cani DS, Gavazzoni M, et al. Impact of disproportionate secondary mitral regurgitation in patients undergoing edge-to-edge percutaneous mitral valve repair. EuroIntervention 2020;16:413-20.

19. Orban M, Karam N, Lubos E, et al; EuroSMR Investigators. Impact of proportionality of secondary mitral regurgitation on outcome after transcatheter mitral valve repair. JACC Cardiovasc Imaging 2020:S1936-878X(20)30620-3.

20. Ben-Shoshan J, Overtchook P, Buithieu J, et al. Predictors of outcomes following transcatheter edge-to-edge mitral valve repair. JACC Cardiovasc Interv 2020;13:1733-48

21. Zanolla L, Zardini P. Selection of endpoints for heart failure clinical trials. Eur J Heart Fail 2003;5:717-23.

22. Anker SD, Schroeder S, Atar D, et al. Traditional and new composite endpoints in heart failure clinical trials: facilitating comprehensive efficacy assessments and improving trial efficiency. Eur J Heart Fail 2016;18:482-9.

23. Fiuzat M, Lowy N, Stockbridge N, et al. Endpoints in heart failure drug development: history and future. JACC Heart Fail 2020;8:42940.

24. Stone GW, Adams DH, Abraham WT, et al; Mitral Valve Academic Research Consortium (MVARC). Clinical trial design principles and endpoint definitions for transcatheter mitral valve repair and replacement: part 2: endpoint definitions a consensus document from the mitral valve academic research consortium. J Am Coll Cardiol 2015;66:308-21.

25. Orban M, Orban M, Lesevic H, et al. Predictors for long-term survival after transcatheter edge-to-edge mitral valve repair. $J$ Interv Cardiol 2017;30:226-33.

26. Braun D, Lesevic H, Orban M, et al. Percutaneous edge-to-edge repair of the mitral valve in patients with degenerative versus functional mitral regurgitation. Catheter Cardiovasc Interv 2014;84:137-46.

27. Öztürk C, Friederich M, Werner N, et al. Single-center five-year outcomes after interventional edge-to-edge repair of the mitral valve. Cardiol J 2019; doi: 10.5603/CJ.a2019.0071.

28. Sürder D, Klersy C, Corti R, et al; Collaborators. Impact of mitral regurgitation aetiology on MitraClip outcomes: the MitraSwiss registry. EuroIntervention 2020;16:e112-20.

29. Dörr O, Walther C, Liebetrau C, et al. Galectin-3 and ST2 as predictors of therapeutic success in high-risk patients undergoing percutaneous mitral valve repair (MitraClip). Clin Cardiol 2018;41:1164-9.

30. Thaden JJ, Malouf JF, Nkomo VT, et al. Mitral valve anatomic predictors of hemodynamic success with transcatheter mitral valve repair. $J$ Am Heart Assoc 2018; 7:e007315.

31. Oguz D, Eleid MF, Dhesi S, et al. Quantitative three-dimensional echocardiographic correlates of optimal mitral regurgitation reduction during transcatheter mitral valve repair. J Am Soc Echocardiogr 2019;32:1426-35.e1.

32. Tabata N, Weber M, Sugiura A, et al. Impact of the leaflet-to-annulus index on residual mitral regurgitation in patients undergoing edgeto-edge mitral repair. JACC Cardiovasc Interv 2019;12:2462-72.

33. Reichart D, Kalbacher D, Rübsamen N, et al. The impact of residual mitral regurgitation after MitraClip therapy in functional mitral regurgitation. Eur J Heart Fail 2020; doi: 10.1002/ejhf.1774.

34. Bozdag-Turan I, Paranskaya L, Birkemeyer R, et al. Percutaneous mitral repair with the MitraClip system in patients with mild-tomoderate and severe heart failure: a single-centre experience. Cardiovasc Ther 2014;32:66-73.

35. Adamo M, Grasso C, Capodanno D, et al. Five-year clinical outcomes after percutaneous edge-to-edge mitral valve repair: Insights from the multicenter GRASP-IT registry. Am Heart J 2019;217:32-41.

36. Paranskaya L, D’Ancona G, Bozdag-Turan I, et al. Early and mid-term outcomes of percutaneous mitral valve repair with the MitraClip®: 
comparative analysis of different EuroSCORE strata. EuroIntervention 2012;8:571-8.

37. Yzeiraj E, Bijuklic K, Tiburtius C, et al. Tricuspid regurgitation is a predictor of mortality after percutaneous mitral valve edge-to-edge repair. EuroIntervention 2017;12:e1817-24.

38. Itabashi $\mathrm{Y}$, Utsunomiya $\mathrm{H}$, Kubo S, et al. Different indicators for postprocedural mitral stenosis caused by single- or multiple-clip implantation after percutaneous mitral valve repair. J Cardiol 2018;71:336-45.

39. Watanabe N, Ogasawara Y, Yamaura Y, et al. Mitral annulus flattens in ischemic mitral regurgitation: geometric differences between inferior and anterior myocardial infarction: a real-time 3-dimensional echocardiographic study. Circulation 2005;112:I458-62.

40. El Sebaie MH, Abdelatti MN, Zarea AA, et al. Assessment of mitral valve geometric deformity in patients with ischemic heart disease using three-dimensional echocardiography. Egypt Heart J 2017;69:13-20.

41. Stolfo D, De Luca A, Morea G, et al. Predicting device failure after percutaneous repair of functional mitral regurgitation in advanced heart failure: Implications for patient selection. Int J Cardiol 2018;257:182-7.

42. Kalbacher D, Schäfer U, V Bardeleben RS, et al. Long-term outcome, survival and predictors of mortality after MitraClip therapy: Results from the German Transcatheter Mitral Valve Interventions (TRAMI) registry. Int J Cardiol 2019;277:35-41.

43. Bannehr M, Kahn U, Okamoto M, et al. Post-procedural tricuspid regurgitation predicts long-term survival in patients undergoing percutaneous mitral valve repair. J Cardiol 2019;74:524-31.

44. Buzzatti N, De Bonis M, Denti P, et al. What is a "good" result after transcatheter mitral repair? Impact of 2+ residual mitral regurgitation. J Thorac Cardiovasc Surg 2016;151:88-96.

45. Rudolph V, Lubos E, Schlüter M, et al. Aetiology of mitral regurgitation differentially affects 2-year adverse outcomes after MitraClip therapy in high-risk patients. Eur J Heart Fail 2013;15:796-807.

46. Azzalini L, Millán X, Khan R, et al. Impact of left ventricular function on clinical outcomes of functional mitral regurgitation patients undergoing transcatheter mitral valve repair. Catheter Cardiovasc Interv 2016;88:1124-33.

47. Baldi C, Citro R, Silverio A, et al. Predictors of outcome in heart failure patients with severe functional mitral regurgitation undergoing MitraClip treatment. Int J Cardiol 2019;284:50-8.

48. Godino C, Scotti A, Taramasso M, et al. Two-year cardiac mortality after MitraClip treatment of functional mitral regurgitation in ischemic and non-ischemic dilated cardiomyopathy. Int J Cardiol 2018;269:33-9.

49. De Bonis M, Taramasso M, Lapenna E, et al. MitraClip therapy and surgical edge-to-edge repair in patients with severe left ventricular dysfunction and secondary mitral regurgitation: mid-term results of a single-centre experience. Eur J Cardiothorac Surg 2016;49:255-62.

50. Bartko PE, Heitzinger G, Arfsten H, et al. Disproportionate functional mitral regurgitation: advancing a conceptual framework to clinical practice. JACC Cardiovasc Imaging 2019;12:2088-90.

51. Keßler M, Pott A, Mammadova E, et al. Atrial fibrillation predicts long-term outcome after transcatheter edge-to-edge mitral valve repair by MitraClip implantation. Biomolecules 2018;8:152.

52. Iliadis C, Baldus S, Kalbacher D, et al. Impact of left atrial diameter on outcome in patients undergoing edge-to-edge mitral valve repair: results from the German TRAnscatheter Mitral valve Interventions (TRAMI) registry. Eur J Heart Fail 2020:22:1202-10.

53. Ledwoch J, Leidgschwendner K, Fellner C, et al. Prognostic impact of left atrial function following transcatheter mitral valve repair. $J$ Am Heart Assoc 2019;8:e011727.

54. Deferm S, Bertrand PB, Verbrugge FH, et al. Atrial functional mitral regurgitation: JACC review topic of the week. J Am Coll Cardiol 2019;73:2465-76.

55. Neuss M, Schau T, Isotani A, et al. Elevated mitral valve pressure gradient after MitraClip implantation deteriorates long-term outcome in patients with severe mitral regurgitation and severe heart failure. JACC Cardiovasc Interv 2017;10:931-9.

56. Boerlage-vanDijk K, Wiegerinck EM, Araki M, et al. Predictors of outcome in patients undergoing MitraClip implantation: an aid to improve patient selection. Int J Cardiol 2015;189:238-43.

57. Puls M, Tichelbäcker T, Bleckmann A, et al. Failure of acute procedural success predicts adverse outcome after percutaneous edge-toedge mitral valve repair with MitraClip. EuroIntervention 2014;9:1407-17.

58. Puls M, Tichelbäcker T, Bleckmann A, et al. Failure of acute procedural success predicts adverse outcome after percutaneous edge-toedge mitral valve repair with MitraClip. EuroIntervention 2014;9:1407-17.

59. Sürder D, Pedrazzini G, Gaemperli O, et al. Predictors for efficacy of percutaneous mitral valve repair using the MitraClip system: the results of the MitraSwiss registry. Heart 2013;99:1034-40.

60. Capodanno D, Adamo M, Barbanti M, et al; GRASP-IT Investigators. Predictors of clinical outcomes after edge-to-edge percutaneous mitral valve repair. Am Heart J 2015;170:187-95.

61. Patzelt J, Zhang W, Sauter R, et al. Elevated mitral valve pressure gradient is predictive of long-term outcome after percutaneous edge-to-edge mitral valve repair in patients with degenerative mitral regurgitation (MR), but not in functional MR. J Am Heart Assoc 2019;8:e11366.

62. Alessandrini H, Kreidel F, Schlüter M, et al. Prognostic implication of post-MitraClip vena contracta area in heart failure patients with functional mitral regurgitation. EuroIntervention 2017;12:1946-53.

63. Taramasso M, Denti P, Latib A, et al. Clinical and anatomical predictors of MitraClip therapy failure for functional mitral regurgitation: single central clip strategy in asymmetric tethering. Int J Cardiol 2015;186:286-8.

64. Kitamura M, Kaneko H, Schlüter M, et al. Predictors of mortality in ischaemic versus non-ischaemic functional mitral regurgitation after successful transcatheter mitral valve repair using MitraClip: results from two high-volume centres. Clin Res Cardiol 2019;108:264-72.

65. Hoit BD. Atrial functional mitral regurgitation: the left atrium gets its due respect. J Am Coll Cardiol 2011;58:1482-4.

66. Kagiyama N, Mondillo S, Yoshida K, Mandoli GE, Cameli M. Subtypes of atrial functional mitral regurgitation: imaging insights into 
their mechanisms and therapeutic implications. JACC Cardiovasc Imaging 2020;13:820-35.

67. Tigges E, Blankenberg S, von Bardeleben RS, et al. Implication of pulmonary hypertension in patients undergoing MitraClip therapy: results from the German transcatheter mitral valve interventions (TRAMI) registry. Eur J Heart Fail 2018;20:585-94.

68. Yucel E, Al-Bawardy R, Bertrand PB. Pulmonary hypertension in patients eligible for transcatheter mitral valve repair: prognostic impact and clinical implications. Curr Treat Options Cardiovasc Med 2019;21:60.

69. Al-Bawardy R, Vemulapalli S, Thourani VH, et al. Association of pulmonary hypertension with clinical outcomes of transcatheter mitral valve repair. JAMA Cardiol 2020;5:47-56.

70. Osteresch R, Diehl K, Kühl M, et al. Impact of right heart function on outcome in patients with functional mitral regurgitation and chronic heart failure undergoing percutaneous edge-to-edge-repair. J Interv Cardiol 2018;31:916-24.

71. Kaneko H, Neuss M, Weissenborn J, Butter C. Prognostic significance of right ventricular dysfunction in patients with functional mitral regurgitation undergoing MitraClip. Am J Cardiol 2016;118:1717-22.

72. Giannini C, Fiorelli F, Colombo A, et al. Right ventricular evaluation to improve survival outcome in patients with severe functional mitral regurgitation and advanced heart failure undergoing MitraClip therapy. Int J Cardiol 2016;223:574-80.

73. Godino C, Salerno A, Cera M, et al. Impact and evolution of right ventricular dysfunction after successful MitraClip implantation in patients with functional mitral regurgitation. Int J Cardiol Heart Vasc 2016;11:90-8.

74. Schueler R, Öztürk C, Sinning JM, et al. Impact of baseline tricuspid regurgitation on long-term clinical outcomes and survival after interventional edge-to-edge repair for mitral regurgitation. Clin Res Cardiol 2017;106:350-8.

75. Al-Hindwan HSA, Silbernagel G, Curio J, et al. The impact of moderate aortic valve disease in patients undergoing MitraClip for severe MR. Clin Hemorheol Microcirc 2020; doi: 10.3233/CH-200818.

76. Geis NA, Puls M, Lubos E, et al. Safety and efficacy of MitraClip ${ }^{\mathrm{TM}}$ therapy in patients with severely impaired left ventricular ejection fraction: results from the German transcatheter mitral valve interventions (TRAMI) registry. Eur J Heart Fail 2018;20:598-608.

77. Gyoten T, Messroghli D, Schenk S, et al. Impact of preinterventional tricuspid regurgitation on outcome of MitraClip therapy in patients with severely reduced ejection fraction. Open Heart 2020;7:e001203.

78. Asch FM, Grayburn PA, Siegel RJ, et al; COAPT Investigators. Echocardiographic outcomes after transcatheter leaflet approximation in patients with secondary mitral regurgitation: the COAPT trial. J Am Coll Cardiol 2019;74:2969-79.

79. Schueler R, Nickenig G, May AE, et al. Predictors for short-term outcomes of patients undergoing transcatheter mitral valve interventions: analysis of 778 prospective patients from the German TRAMI registry focusing on baseline renal function. EuroIntervention 2016;12:50814.

80. Triantafyllis AS, Kortlandt F, Bakker AL, et al. Long-term survival and preprocedural predictors of mortality in high surgical risk patients undergoing percutaneous mitral valve repair. Catheter Cardiovasc Interv 2016;87:467-75.

81. Kyhse-Andersen J, Schmidt C, Nordin G, et al. Serum cystatin C, determined by a rapid, automated particle-enhanced turbidimetric method, is a better marker than serum creatinine for glomerular filtration rate. Clin Chem 1994;40:1921-6.

82. Devarajan P. Neutrophil gelatinase-associated lipocalin: a promising biomarker for human acute kidney injury. Biomark Med 2010;4:265-80.

83. Sims JR, Reeder GS, Guerrero M, et al. Characteristics and outcomes of patients with normal left atrial pressure undergoing transcatheter mitral valve repair. Heart 2020;106:898-903.

84. Gotzmann M, Sprenger I, Ewers A, Mügge A, Bösche L. One-year outcome of percutaneous mitral valve repair in patients with severe symptomatic mitral valve regurgitation. World J Cardiol 2017;9:39-46.

85. Buccheri S, Capodanno D, Barbanti M, et al. A risk model for prediction of 1-year mortality in patients undergoing MitraClip implantation. Am J Cardiol 2017;119:1443-9.

86. Ailawadi G, Lim DS, Mack MJ, et al. One-year outcomes after MitraClip for functional mitral regurgitation. Circulation 2019;139:37-47.

87. Kalbacher D, Schäfer U, von Bardeleben RS, et al. Impact of tricuspid valve regurgitation in surgical high-risk patients undergoing MitraClip implantation: results from the TRAMI registry. EuroIntervention 2017;12:e1809-16.

88. Matsumoto T, Nakamura M, Yeow WL, et al. Impact of pulmonary hypertension on outcomes in patients with functional mitral regurgitation undergoing percutaneous edge-to-edge repair. Am J Cardiol 2014;114:1735-9.

89. Dörr $\mathrm{O}$, Walther $\mathrm{C}$, Liebetrau $\mathrm{C}$, et al. Evaluation of cystatin $\mathrm{C}$ and neutrophil gelatinase-associated lipocalin as predictors of mortality in patients undergoing percutaneous mitral valve repair (MitraClip). Clin Cardiol 2018;41:1474-9.

90. Keßler M, Seeger J, Muche R, et al. Predictors of rehospitalization after percutaneous edge-to-edge mitral valve repair by MitraClip implantation. Eur J Heart Fail 2019;21:182-92. 